\title{
Comparison of Matrix Pencil Extracted Features in Time Domain and in Frequency Domain for Radar Target Classification
}

\author{
Mahmoud Khodjet-Kesba, ${ }^{1,2,3}$ Khalil El Khamlichi Drissi, ${ }^{1,2}$ Sukhan Lee, ${ }^{3}$ \\ Kamal Kerroum, ${ }^{1,2}$ Claire Faure, ${ }^{1,2}$ and Christophe Pasquier ${ }^{1,2}$ \\ ${ }^{1}$ Clermont Université, Université Blaise Pascal, BP 10448, 63000 Clermont Ferrand, France \\ ${ }^{2}$ CNRS, UMR 6602, Institut Pascal, 63177 Aubière, France \\ ${ }^{3}$ ISRI, 300 Cheoncheon-Dong, Jangan-Gu, Suwon, Gyeonggi-Do 440-746, Republic of Korea \\ Correspondence should be addressed to Mahmoud Khodjet-Kesba; khomah84@gmail.com
}

Received 5 November 2013; Accepted 24 December 2013; Published 4 February 2014

Academic Editor: Tayeb A. Denidni

Copyright (C) 2014 Mahmoud Khodjet-Kesba et al. This is an open access article distributed under the Creative Commons Attribution License, which permits unrestricted use, distribution, and reproduction in any medium, provided the original work is properly cited.

\begin{abstract}
Feature extraction is a challenging problem in radar target identification. In this paper, we propose a new approach of feature extraction by using Matrix Pencil Method in Frequency Domain (MPMFD). The proposed method takes into account not only the magnitude of the signal, but also its phase, so that all the physical characteristics of the target will be considered. With this method, the separation between the early time and the late time is not necessary. The proposed method is compared to Matrix Pencil Method in Time Domain (MPMTD). The methods are applied on UWB backscattered signal from three canonical targets (thin wire, sphere, and cylinder). MPMFD is applied on a complex field (real and imaginary parts of the signal). To the best of our knowledge, this comparison and the reconstruction of the complex electromagnetic field by MPMFD have not been done before. We show the effect of the two extraction methods on the accuracy of three different classifiers: Naïve bayes (NB), K-Nearest Neighbor (K-NN), and Support Vector Machine (SVM). The results show that the accuracy of classification is better when using extracted features by MPMFD with SVM.
\end{abstract}

\section{Introduction}

In the last fifteen years, the interest in ultra wideband systems has grown rapidly. One of several applications of the UWB is automatic target classification in intelligent vehicles. The UWB technique has the advantage to be used for localization, target identification, and communication between vehicles. The scope of this paper focuses on using UWB radar for automatic target classification. UWB radar uses very short duration pulses resulting in occupying a very wide band in frequency domain. This technique is defined by the Federal Communication Commission (FCC) as it possesses at least one of the following characteristics [1]:

(i) the spectrum width $B$ of radiated signals determined at a level of $-10 \mathrm{~dB}$ relative to the spectrum maximum is at least $500 \mathrm{MHz}$; (ii) the relative bandwidth $B / f_{0}$ at a level of $-10 \mathrm{~dB}$ is not less than 0.2 (where $f_{0}$ is the spectrum's center frequency).

In intelligent vehicles, the UWB radar can be a complementary solution to identify obstacles as pedestrians, animals, vehicles, and so forth. The aim is to classify automatically the target in specified zone. The identification of the target will be realized by the comparison between the deduced target properties and the different target features already recorded in a database. Selection of proper feature vectors is crucial to the success of target identification. Using the complex natural resonances (CNRs) as features has been receiving much attention in the last decade. Various techniques have been developed to extract poles, such as the E-pulse approach [2], the Trufts and Kumaresan method [3], 
and the high resolution methods like Matrix Pencil Method [4]. All these methods are applied to the late time part of the signal based on the Singularity Expansion Method (SEM). This part of signal arises from resonance phenomena of the target. It depends, at least theoretically, on the target geometry and its physical properties. Thus, it is independent of the aspect and polarization of the excitation source [5]. However, the automatic determination of the late time is not an easy task [6]. CNRs can be extracted in the frequency domain by using the Cauchy method. In [7], this method is applied to compute the natural poles of an object in the frequency domain; however, in real time applications, this method is inconvenient.

In this paper, we propose an innovative approach of feature extraction in frequency domain. The proposed method takes into account not only the magnitude of the signal in frequency domain but also its phase. Therefore, all the physical characteristics of the target are taken into account. The physical and geometrical characteristics of the considered object and the incident waveform impact clearly the signatures of the treated targets and contribute efficiently to the classification of the objects. The separation between the early time and the late time is not necessary by applying this method.

Two types of features are compared in this paper. First, Matrix Pencil Method in Time Domain (MPMTD) is applied on the late time part of the backscattered signal to extract CNRs. Second, Matrix Pencil Method in Frequency Domain (MPMFD) is applied on the complex frequency field to extract features. In this case the early time behavior is taken into account, which is dominated by localized specular reflections from target scattering centers.

Simulations are conducted by using three metallic canonical objects: thin wire, sphere, and cylinder. Two databases are built for the comparison: the first database contains features extracted by MPMTD, and the second one contains features extracted by MPMFD. The accuracy of the classification by using different classifiers [8] such as Naïve bayes (NB), K-Nearest Neighbor (K-NN), and Support Vector Machine (SVM) has been determined.

The paper is organized as follows. Section 2 presents the feature extraction in time domain and in frequency domain. Section 3 introduces the classification methods used in this paper, and in Section 4, the simulation results and discussion are presented. Finally, Section 5 gives our conclusions related to this study.

\section{Feature Extraction}

2.1. The Signal in Time Domain (SEM). When a target is illuminated by ultra wideband signals, the scattered transient response in the time domain consists of an impulsive waveform part (early time) followed by series of damped oscillations (late time). The early time response arises from the direct reflection of the incident wave on the object surface. In the late time, the oscillating part arises from resonance phenomena of the target. The resonances can be separated into internal and external modes [9]. The internal resonances are caused by the internal waves experiencing multiple internal reflections, whereas the external resonances are caused by the surface creeping waves. In the case where targets are perfect conductors, resonances occur outside the object and correspond only to external modes.

Baum [10] proposed that the late time response of a specific target can be expressed as series of damped exponentials of the following form:

$$
h(t)=\sum_{i} R_{i} e^{S_{i} t}
$$

(i) $R_{i}$ is the residue associated with each natural pole,

(ii) $S_{i}$ is the $i$ th pole of the target: $S_{i}=\sigma_{i}+j \omega_{i}$,

(iii) $\sigma_{i}$ is the damping factor,

(iv) $\omega_{i}=j 2 \pi f_{i}$ with $f_{i}$ being the natural resonant frequency.

2.2. Matrix Pencil Method in Time Domain. The Matrix Pencil Method will be applied on the late time instants of a target illuminated by a broadband electromagnetic wave. Matrix Pencil Method is a technique of estimation which describes the signal as a sum of finite complex exponentials:

$$
y(t)=h(t)+n(t)=\sum_{i=1}^{M} R_{i} e^{S_{i} t}+n(t)
$$

where $h(t)$ is the signal, $n(t)$ is the noise, and $M$ is the number of poles.

After the sampling procedure, the observed response (consisting of $N$ samples) can be written as follows:

$$
y\left(k T_{s}\right)=h\left(k T_{s}\right)+n\left(k T_{s}\right) \approx \sum_{i=1}^{M} R_{i} z_{i}^{k}+n\left(k T_{s}\right),
$$

where $T_{s}$ is the sampling period and $k=0, \ldots, N-1$, with:

$$
\begin{gathered}
R_{i}=\left|a_{i}\right| e^{j \varphi_{i}}, \quad i=1,2, \ldots, M, \\
z_{i}=e^{S_{i} T_{s}}=e^{\left(\sigma_{i}+j \omega_{i}\right) T_{s}}, \quad i=1,2, \ldots, M .
\end{gathered}
$$

MPMTD is applied following [4] to extract $z_{i}$ and $R_{i}$. The real and imaginary parts $\left(\sigma_{i}\right.$ and $\left.\omega_{i}\right)$ of the poles are the features used for automatic target classification.

In our work, to find the beginning of the late time we have used the following expression [11]:

$$
T_{L}=T_{b}+2 T_{\mathrm{tr}}+T_{p},
$$

where $T_{b}$ is the time when incident wave strikes the leading edge of the target, $T_{\mathrm{tr}}$ is the maximum transit time of the target, and $T_{p}$ is the pulse width of the transmitted waveform.

To use the expression (6), we need to know in advance the size of the target, so it is inconvenient in automatic target classification. Another method can be applied based on using a sliding window and observing the stability of the poles after moving the beginning of the window. However, in Section 4, we will see that this method is not accurate for less resonant objects as the sphere and the cylinder. 
2.3. The Signal in Frequency Domain. In frequency domain, the scattered fields $E_{\nu}(\omega, r)$ can be expressed as follows [12]:

$$
E_{v}(\omega, r)=\sum_{n=1}^{\infty} A_{\nu n}(\omega, r) \exp \left(-j \omega t_{n}\right),
$$

where $v$ is any scattering field component of an orthogonal coordinate system (polarization), $\omega$ is the angular frequency, $A_{\nu n}(\omega, r)$ is the complex and frequency-dependent amplitude of the $n$th scattering center depending on the scattering mechanism, $r$ is the far-field position, and $t_{n}$ is the time delay between the observer and the $n$th scattering center. The time dependence $\exp (j \omega t)$ and the $v$ are dropped for convenience throughout.

The following approximation [13] is used:

$$
A_{n}(\omega, r) \approx a_{n}(r) e^{\left(\gamma_{n} \omega\right)},
$$

where $a_{n}(r)$ is the amplitude and $\gamma_{n}$ is the phase which provides an approximate match between $A_{n}(\omega, r)$ and the exponential model. This approximation can only be met over a relatively narrow bandwidth. After using (8) in (7) and the sampling procedure, the frequency response $Y\left(k \omega_{s}\right)$ will be expressed as

$$
\begin{array}{r}
Y\left(k \omega_{s}\right) \approx \sum_{n=1}^{M_{f}} \widehat{a}_{n}(r) e^{\left(\gamma_{n} k \omega_{s}-j k \omega_{s} t_{n}\right)}+n\left(k \omega_{s}\right), \\
k=0, \ldots, N_{f}-1,
\end{array}
$$

where $\omega_{s}$ is the frequency sampling, $N_{f}$ is the number of frequency samples, $M_{f}$ is the number of measurable wavefronts, $\widehat{a}_{n}(r)$ is the complex amplitude, and $n\left(k \omega_{s}\right)$ is the additive noise.

2.4. Matrix Pencil Method in Frequency Domain. The MPMFD is applied on the frequency response $Y\left(k \omega_{s}\right)$ :

$$
Y\left(k \omega_{s}\right) \approx \sum_{n=1}^{M_{f}} \widehat{a}_{n}(r) x_{n}+n\left(k \omega_{s}\right)
$$

with

$$
x_{n}=e^{\left(\gamma_{n} k \omega_{s}-j k \omega_{s} t_{n}\right)} .
$$

It is performed in a few steps.

First, a Hankel's matrix is constructed:

$$
\widehat{Y}=\left(\begin{array}{cccc}
Y(0) & Y(1) & \cdots & Y(L) \\
Y(1) & Y(2) & \ldots & Y(L+1) \\
\vdots & \vdots & \vdots & \vdots \\
Y\left(N_{f}-L-1\right) & Y\left(N_{f}-L\right) & \cdots & Y\left(N_{f}-1\right)
\end{array}\right) \text {, }
$$

where $\widehat{Y}$ is dimensions $\left(N_{f}-L\right) \times(L+1)$ and $L$ is the Pencil parameter. The choice of $L$ is important to reduce numerical noises. The best choice is [4]

$$
\frac{N_{f}}{2} \leq L \leq \frac{2 N_{f}}{3} \text {. }
$$

Next, a singular value decomposition of the matrix is carried out as

$$
[\widehat{Y}]=[U] \cdot[\Sigma] \cdot[V]^{H},
$$

where $[U]$ of dimension $\left(N_{f}-L\right) \times\left(N_{f}-L\right)$ and $[V]$ of dimension $(L+1) \times(L+1)$ are unitary matrix of eigenvectors, while $[\Sigma]$ of dimension $\left(N_{f}-L\right) \times(L+1)$ is a diagonal matrix of singular values $\sigma_{i}$, and the superscript " $H$ " denotes the conjugate transpose. The number of significant poles $M_{f}$ should be estimated by the ratio of singular values to the largest one:

$$
\frac{\sigma_{M_{f}}}{\sigma_{1}} \approx 10^{-p},
$$

where $p$ is the number of significant decimal digits in the data. In our work, the optimal $p$ which allows a good reconstruction of the signal was found to be equal to 2 . The value of $M_{f}$ should verify the dual condition:

$$
M_{f} \leq L \leq N_{f}-M_{f}
$$

Next, the filtered matrix $\left[V_{M_{f}}\right]$ of dimension $(L+1) \times\left(M_{f}\right)$ corresponding to the first $M_{f}$ vectors of $[V]$ is considered:

$$
\left[V_{M_{f}}\right]=\left[v_{1}, v_{2}, \ldots, v_{M_{f}}\right]
$$

From $\left[V_{M_{f}}\right]$ we generate $\left[V_{M_{f 1}}\right]$ by deleting the last row of $\left[V_{M_{f}}\right]$ and $\left[V_{M_{f 2}}\right]$ by deleting the first row of $\left[V_{M_{f}}\right]$. The eigenvalue of lower matrix $\operatorname{rank}\left\{\left[V_{M_{f 2}}\right]^{H}-\lambda\left[V_{M_{f 1}}\right]\right\}$ provides the estimation of $x_{n}$.

Once $M_{f}$ and $x_{n}$ are known, the complex amplitudes $\widehat{a}_{n}(r)$ are solved by using the following least square equation:

$$
\left(\begin{array}{c}
Y(0) \\
Y(1) \\
\vdots \\
Y\left(N_{f}-1\right)
\end{array}\right)=\left(\begin{array}{cccc}
1 & 1 & \cdots & 1 \\
x_{1} & x_{2} & \cdots & x_{M_{f}} \\
\vdots & \vdots & \vdots & \vdots \\
x_{1}^{\left(N_{f}-1\right)} & x_{2}^{\left(N_{f}-1\right)} & \cdots & x_{M_{f}}^{\left(N_{f}-1\right)}
\end{array}\right)
$$

$$
\times\left(\begin{array}{c}
\widehat{a}_{1} \\
\widehat{a}_{2} \\
\vdots \\
\widehat{a}_{M_{f}}
\end{array}\right)
$$

In frequency domain, the time delay $t_{n}$ and the phase $\gamma_{n}$ are used as features for automatic target classification.

\section{Classification Methods}

Supervised classification algorithms enable one to assign a class label for each input example [14]. Given a training data set of the form $\left(x_{i}, c_{i}\right)$, where $x_{i} \in \mathfrak{R}^{n}$ is the $i$ th example and $c_{i} \in\{1, \ldots, K\}$ is the $i$ th class label, the main objective is to find a learning model $\Lambda$ that corresponds to $\Lambda\left(x_{i}\right)=c_{i}$ for 
new unknown examples. Any classification process has two phases.

(i) Training or learning phase: in this phase the learning algorithm is applied on a subset of the dataset, called training data. This results in a trained model.

(ii) Test phase: in this phase another subset of the data, called test data, is evaluated using the model created in the training phase.

To calculate the performance of a learning model with a better generalization, validation has been applied on a given problem. The idea is to have a subset of the dataset, called validation set which is not included in the training set, and is considered as a test set. One of the most powerful validation techniques is the cross-validation; with this method, the performance of an algorithm on an input dataset is averaged over several rounds of evaluation.

We have used the leave-one-out cross-validation. Using this method, we split the data set of size $m$ into $m$ partitions of size 1. Each partition is used for testing only once, whereas the remaining partitions are used for training. The estimation of the overall accuracy is calculated as an average of the $m$ individual accuracy measures. In our work, we have used 8 examples for each one of the 3 canonical targets, so: $m=3 *$ $8=24$.

3.1. Nä̈ve Bayes. This classifier is based on Bayes' theorem and the maximum a posteriori hypothesis [15]. Let $x=$ $\left(x_{1}, \ldots, x_{N}\right)$ be an $N$-dimensional instance which has no class label. Our goal is to build a classifier to predict its unknown class label. Let $C=\left\{C_{1}, \ldots, C_{K}\right\}$ be the set of the class labels. $P\left(C_{k}\right)$ is the prior probability of $C_{k}(k=1, \ldots, K)$; $P\left(x \mid C_{k}\right)$ is the conditional probability of the evidence $x$ if the hypothesis $C_{K}$ is true. It is necessary to assess the class maximizing $P\left(C_{k} \mid x\right)$. The class $C_{k}$ which maximizes $P\left(C_{k} \mid\right.$ $x)$ is called the maximum a posteriori hypothesis. By using Bayes' theorem, we obtain

$$
P\left(C_{k} \mid x\right)=\frac{P\left(x \mid C_{k}\right) P\left(C_{k}\right)}{P(x)} .
$$

A naive Bayes classifier assumes that the value of a particular feature of a class is unrelated to the value of any other feature, so that

$$
P\left(x \mid C_{k}\right)=\prod_{j=1}^{N} P\left(x_{j} \mid C_{k}\right) .
$$

3.2. K-NN. K-Nearest Neighbor is based on the principle that the instances within a dataset will generally exist in close proximity to other instances having similar properties. If the instances are tagged with a classification label, then the value of the label of an unclassified instance can be determined by observing the class of its nearest neighbors [16]. To classify an unknown example, the distance from that example to every other training example is measured. Usually the Euclidean distance criterion is used. A Euclidean distance between any pair $x_{1}=\left(x_{1,1}, \ldots, x_{1, k}\right)$ and $x_{2}=\left(x_{2,1}, \ldots, x_{2, k}\right)$ of instances is defined as

$$
d\left(x_{1}, x_{2}\right)=\sqrt{\sum_{j=1}^{k}\left(x_{1, j}-x_{2, j}\right)^{2}} .
$$

3.3. Support Vector Machine. Support Vector Machine (SVM) maps the input vectors to a higher dimensional space where a maximal separating hyper plane is constructed [17]. Two parallel hyper planes are constructed on each side of the hyper plane separating the data. The maximum distance between the parallel planes is known as the margin. SVM maximizes the margin and thereby creates the largest possible distance between the separating hyper plane and the examples in the training set on either side of it.

Given a training set of sample-label pairs $\left(x_{i}, c_{i}\right)$, with features $x$, labels $c$, and $i=1, \ldots, K$, the Support Vector Machines are constructed from the following mathematical optimization procedure:

$$
\begin{aligned}
& \underset{w, b, \xi}{\operatorname{minimize}}\left[\frac{1}{2}\left(w^{T} w\right)+C \sum_{i=1}^{K} \xi_{i}\right] \\
& \text { subject to } \quad c_{i}\left(w^{T} \phi\left(x_{i}\right)+b\right) \geq 1-\xi_{i}, \quad \xi_{i} \geq 0 .
\end{aligned}
$$

Here $w$ is the decision plane orientation vector, $b$ is the bias, $\xi_{i}$ represents the margin slack variable, $\phi$ is the mapping function, and $C$ is the penalty parameter of the error term.

SVM has been primarily designed for binary classification problems, but it can also be used in multiclassification problems. Here, we use the so-called "one versus the rest" approach. In this work, a Radial Basis Function (RBF) kernel is implemented, where its parameters are optimized by means of a grid search.

\section{Simulation Results}

4.1. Representation of the Signals in Time and Frequency Domains. To compute the free-space backscattered fields of the targets, electromagnetic commercial tool (TIME-FEKO) has been used [18]. A Gaussian pulse is used as an excitation plane wave; the incidence is normal. In the time domain, the expression of the Gaussian pulse waveform is given by

$$
g(t)=A e^{-\left(\left(t-t_{0}\right) / \sigma\right)^{2}},
$$

where $A$ stands for the amplitude and $\sigma$ for the width of the Gaussian pulse. In our simulations we have set $A=1, t_{0}=$ $10 \mathrm{~ns}$, and $\sigma=0.45455 \cdot 10^{-9} \mathrm{~s}^{-1}$.

$\sigma$ is chosen such that [18] the upper frequency $f_{\max } \approx$ $(4 / \pi \sigma) \sqrt{\ln \sqrt{2}} \approx 1.7 \mathrm{GHz}$. This frequency is chosen by making a compromise between the calculation times and having the radiation of the targets in resonance and optical regions. Note that the frequency band used in our work for target classification is relatively narrow so that the approximation (8) will be valid.

With the TIME-FEKO program, electromagnetic fields are given in the time domain. It is based on the FEKO 

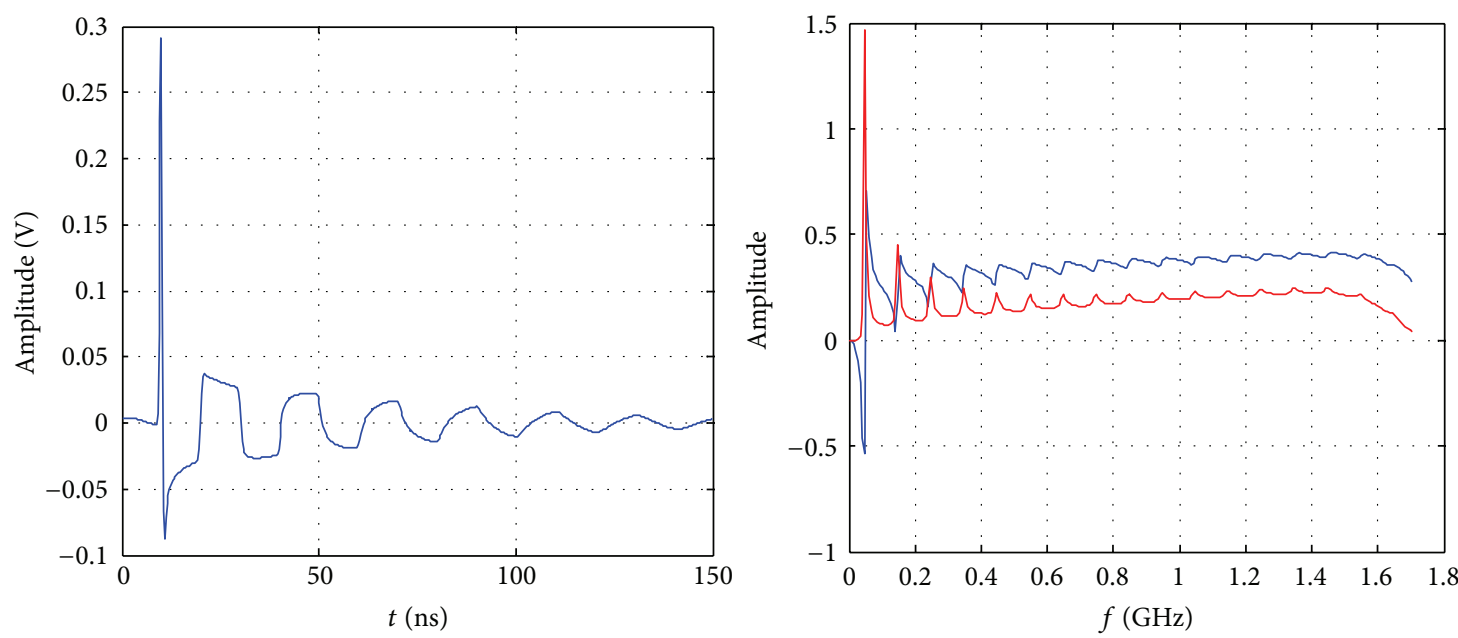

(a) Thin wire (length $=3 \mathrm{~m}$ and radius $=0.0025 \mathrm{~m}$ )
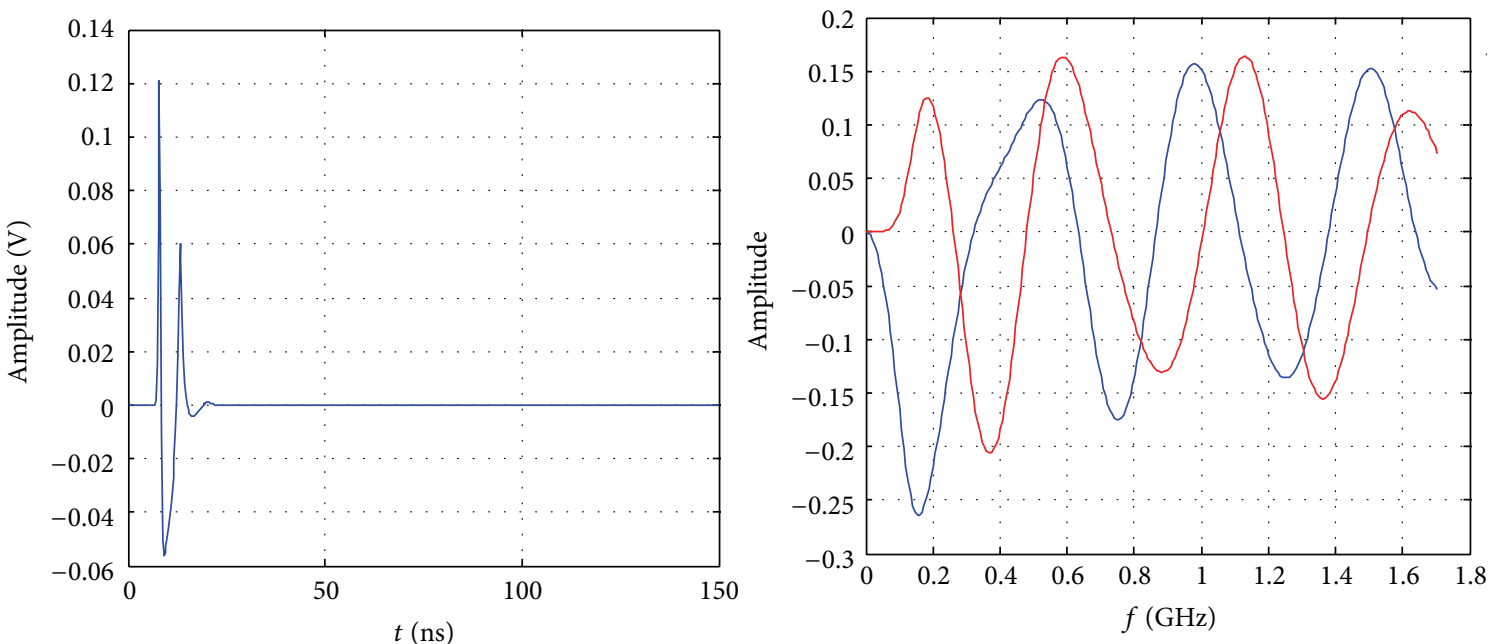

(b) Sphere (radius $=0.3 \mathrm{~m})$
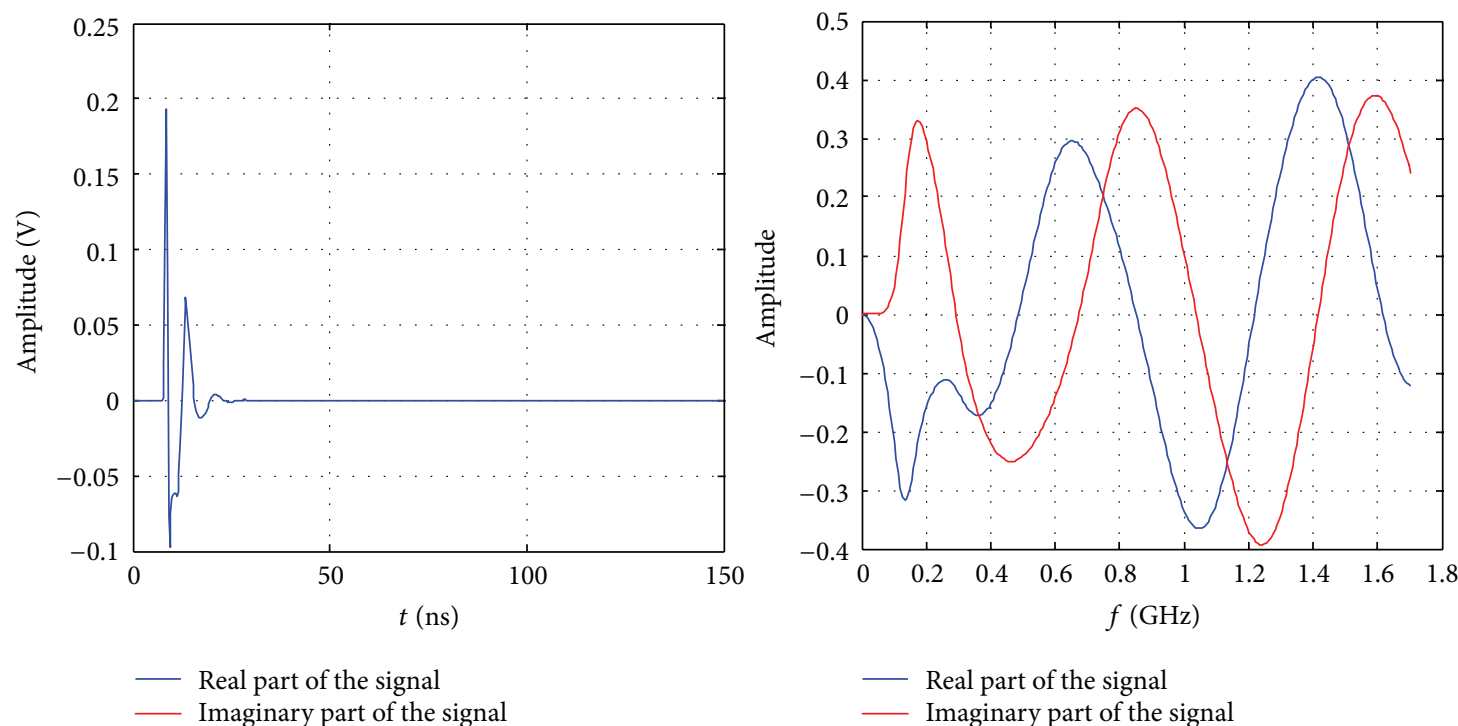

(c) Cylinder (length $=0.6 \mathrm{~m}$ and radius $=0.2 \mathrm{~m}$ )

FIGURE 1: The backscattered fields from the three canonical objects in time domain (left) and in frequency domain (right). 


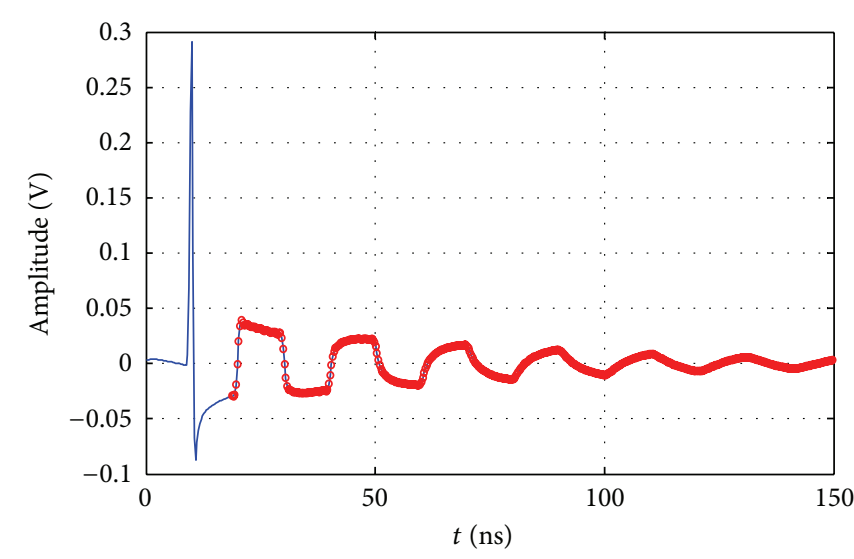

(a) Thin wire (length $=3 \mathrm{~m}$ and radius $=0.0025 \mathrm{~m}$ )

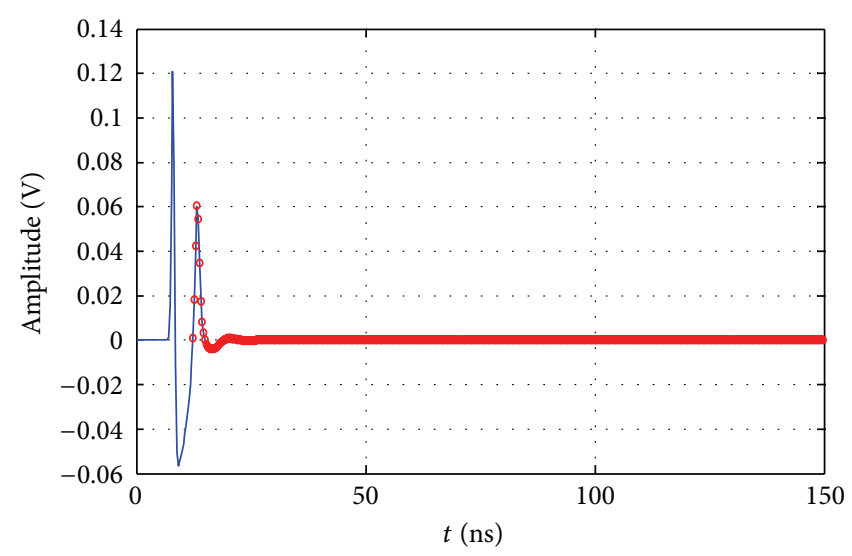

(b) Sphere (radius $=0.3 \mathrm{~m})$

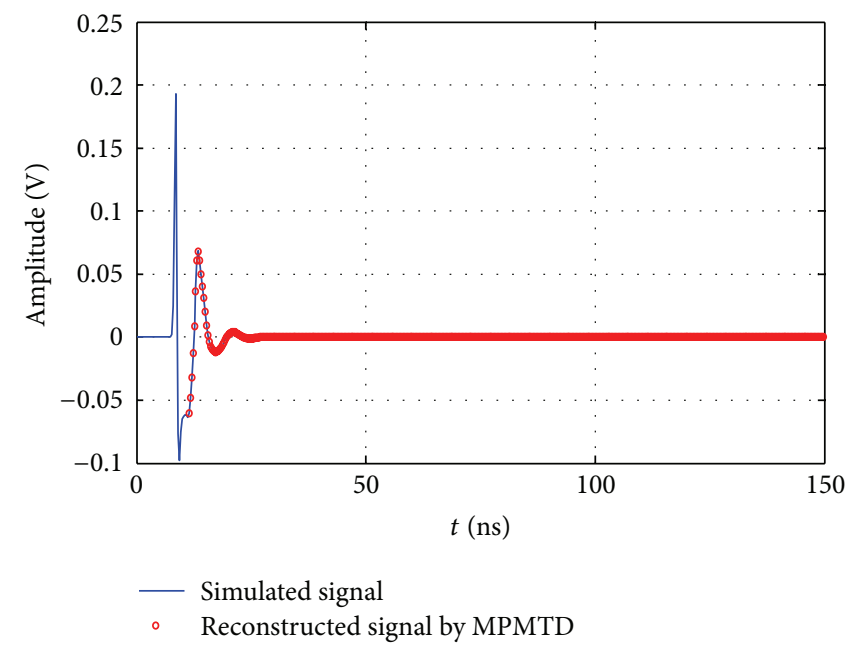

(c) Cylinder (length $=0.6 \mathrm{~m}$ and radius $=0.2 \mathrm{~m}$ )

FIGURE 2: Simulated signal compared with reconstructed signal by MPMTD for a sphere (radius $=0.3 \mathrm{~m}$ ).

program that does the relevant calculations in the frequency domain and an IFFT algorithm that transforms the data to the time domain. The number of frequency samples is chosen to be at 256 .

The backscattered field is given in the time domain without considering the factor $e^{-j k R} / R$.

To apply the MPMFD, we work with the frequency response, which can be obtained from the temporal response by transforming it to the frequency domain by means of the fast Fourier transformed (FFT) technique and dividing it by the FFT of the Gaussian incident pulse.

Figure 1 shows an example from FEKO of the backscattered fields from a thin wire $(l=3 \mathrm{~m}, r=0.0025 \mathrm{~m})$, a sphere $(r=0.3 \mathrm{~m})$, and a cylinder $(l=0.6 \mathrm{~m}, r=0.2 \mathrm{~m})$ in time and frequency domains. The contribution of the late time is very small for less resonant objects. We can see from the figures in the time domain that the wire is very resonant in comparison to the sphere and the cylinder.

4.2. Results from MPMTD. In this section, we analyze the late time part of the temporal response by applying MPMTD.
TABLE 1: Features extracted by MPMTD from a thin wire $(l=3 \mathrm{~m})$, a sphere $(r=0.3 \mathrm{~m})$, and a cylinder $(l=0.6 \mathrm{~m}, r=0.2 \mathrm{~m})$ (poles $\left.\times 10^{9}\right)$.

\begin{tabular}{lccc}
\hline & Thin wire & Sphere & Cylinder \\
\hline$P_{1,2}$ & $-0.080 \mp 3.409 j$ & $-1.859 \mp 4.502 j$ & $-1.313 \mp 5.250 j$ \\
$P_{3,4}$ & $-0.053 \mp 2.791 j$ & $-1.401 \mp 1.729 j$ & $-1.248 \mp 3.858 j$ \\
$P_{5,6}$ & $-0.044 \mp 2.166 j$ & $-0.438 \mp 0.843 j$ & $-0.520 \mp 1.690 j$ \\
$P_{7,8}$ & $-0.038 \mp 1.541 j$ & 0 & $-0.339 \mp 0.849 j$ \\
$P_{9,10}$ & $-0.031 \mp 0.917 j$ & 0 & 0 \\
$P_{11,12}$ & $-0.018 \mp 0.297 j$ & 0 & 0 \\
\hline
\end{tabular}

Table 1 shows the extracted features by MPMTD from a thin wire $(l=3 \mathrm{~m})$, a sphere $(r=0.3 \mathrm{~m})$, and a cylinder $(l=$ $0.6 \mathrm{~m}, r=0.2 \mathrm{~m})$. Features extracted by MPMTD are complex conjugates because the signal is real in time domain. We used the expression (6) to find the beginning of the late time.

In Figure 2, the reconstructed late time signal with MPMTD compared to the simulated one by Time-Feko of the three examples is depicted. The signal is well reconstructed by the MPMTD. 


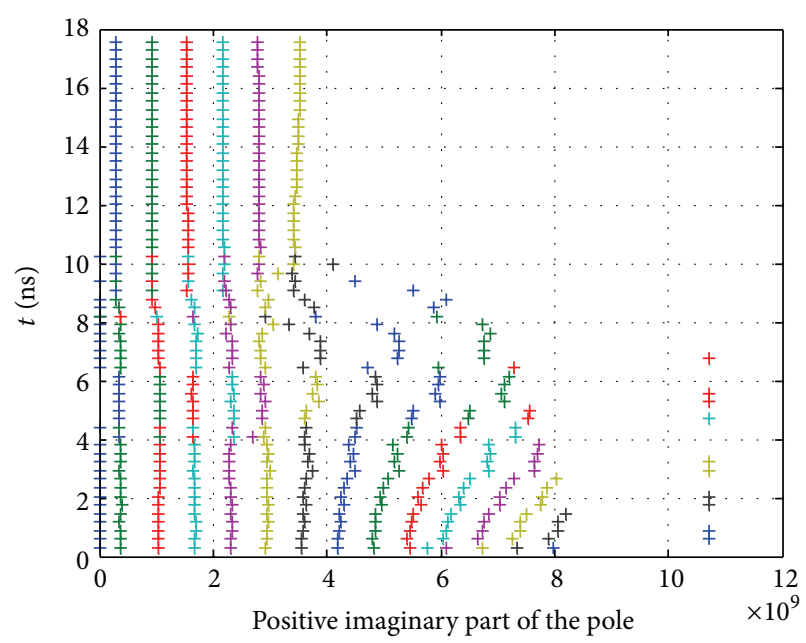

(a) Thin wire (length $=3 \mathrm{~m}$ )

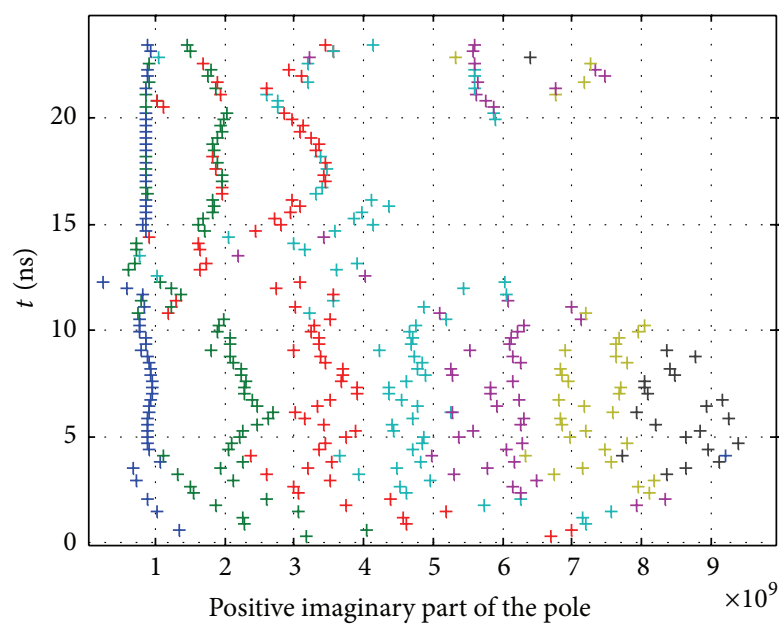

(b) Sphere (radius $=0.3 \mathrm{~m}$ )

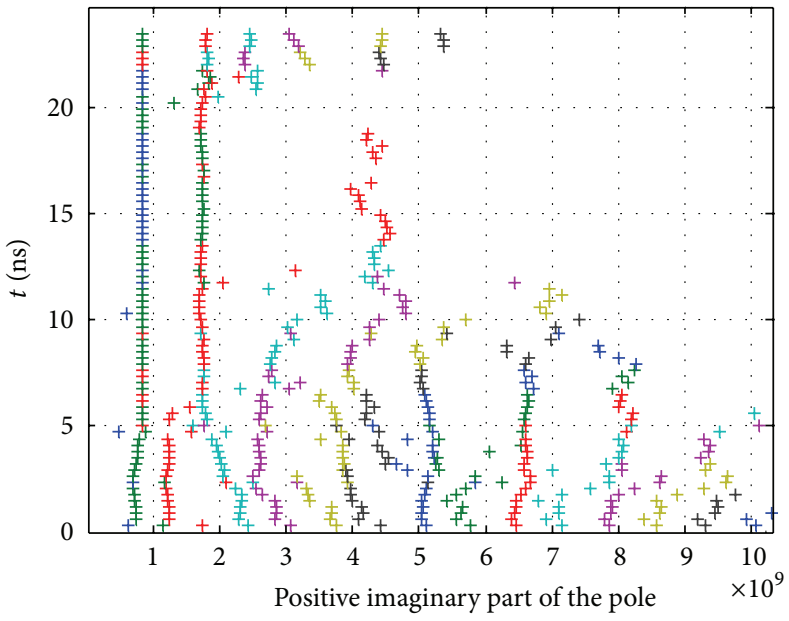

(c) Cylinder (length $=0.6 \mathrm{~m}$ and radius $=0.2 \mathrm{~m}$ )

FIGURE 3: Variation of positive imaginary part of the poles with sliding time.

By moving the beginning of the sliding window through the entire signal by small time steps and by applying MPMTD to the sampled data, we represent the positive imaginary part of the poles as a function of $t_{i}\left(t_{i}\right.$ is the beginning of the late time) in Figure 3. In Figure 3(a) corresponding to the thin wire, we can see the 6 positive imaginary parts of the poles shown in Table 1 at around $t_{i}=10 \mathrm{~ns}$, which corresponds to the beginning of the late time.

The convergence to the natural resonances is stable in the thin wire when $t_{i}$ is shifted in time (the stability is observed in the different wires that we have used), which is not the case for less resonant objects as the sphere and the cylinder. Only the first and second poles are stable (Figures 3(b)-3(c)). The instability of the convergence to the natural resonances is due to the small contribution of the late time part of the signal.

4.3. Results from MPMFD. Here, it is the frequency response (Figure 1) which is analyzed by applying MPMFD.
Table 2, shows the extracted features with MPMFD from the same targets: the thin wire $(l=3 \mathrm{~m}, r=0.0025 \mathrm{~m})$, the sphere $(r=0.3 \mathrm{~m})$, and the cylinder $(l=0.6 \mathrm{~m}, r=0.2 \mathrm{~m})$.

With the poles shown in Table 2, the real and imaginary parts of the signal have been reconstructed in Figure 4 for the three objects. The obtained results agree with the simulated signal.

In frequency domain, all the physical characteristics of the objects are taken into account. The real and imaginary parts of the signals are used to identify the objects.

In Figure 2 we can see the weak contribution of the late time signal in comparison to the contribution of the signal in the frequency domain which takes the early time into consideration (Figure 4). The late-time signature for most realistic targets is usually very weak and heavily corrupted by noise.

4.4. Classification Results. The databases have been built by using 8 examples of different sizes for each perfectly electric 


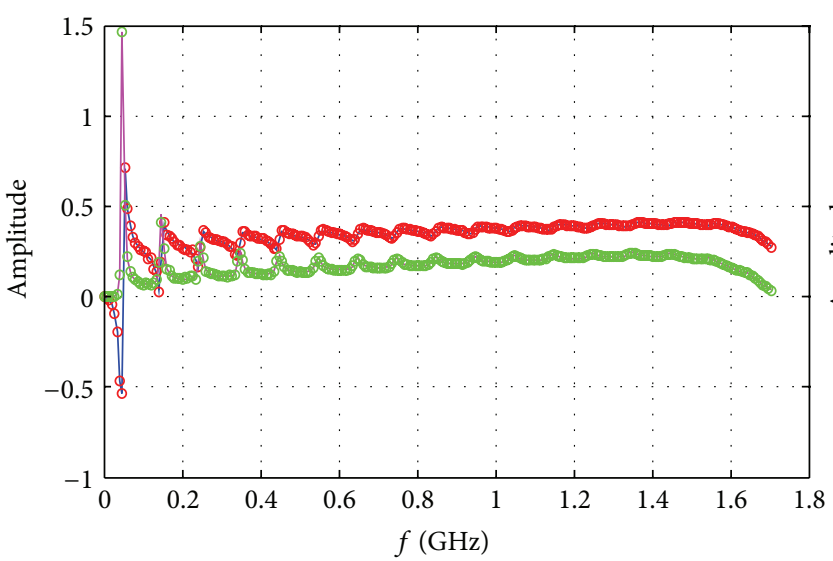

(a) Thin wire (length $=3 \mathrm{~m}$ and radius $=0.0025 \mathrm{~m}$ )

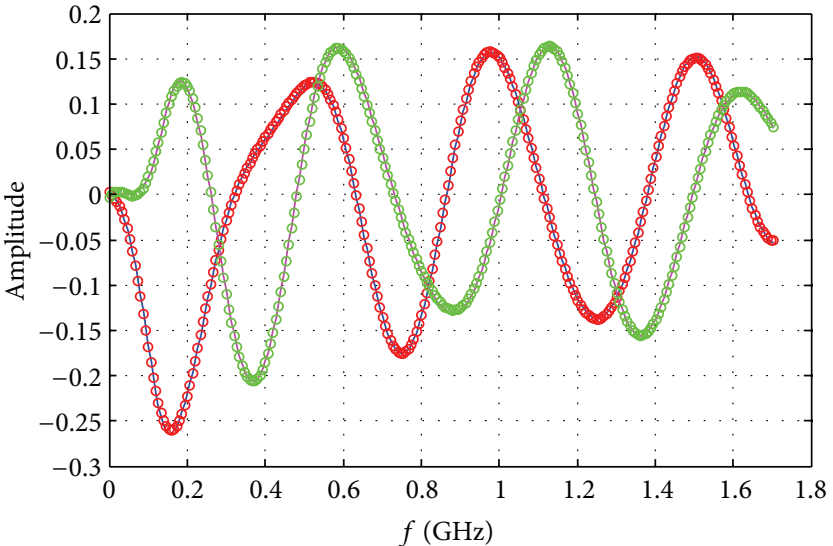

(b) Sphere (radius $=0.3 \mathrm{~m})$

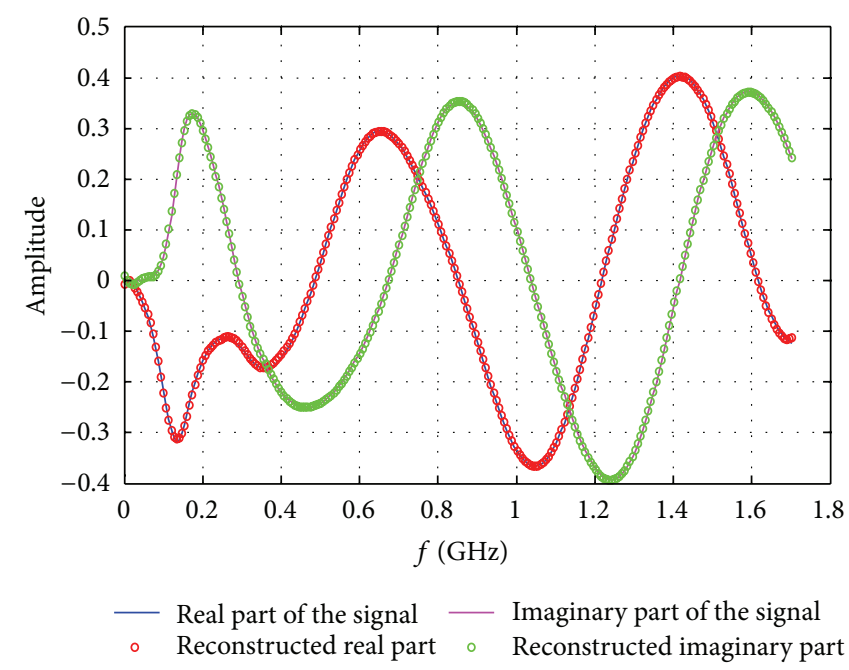

(c) Cylinder (length $=0.6 \mathrm{~m}$ and radius $=0.2 \mathrm{~m}$ )

FigURE 4: Simulated signal compared with reconstructed signal by MPMFD for a sphere (radius $=0.3 \mathrm{~m}$ ).

conducting target: thin wire (length: $l=1,1.3,1.5,1.7,2,2.4$, $2.5,3 \mathrm{~m}$, and radius: $r=0.0025 \mathrm{~m}$ for all the wires), sphere (radius: $r=0.15,0.2,0.28,0.3,0.35,0.4,0.45$, and $0.5 \mathrm{~m}$ ), and cylinder $(l=0.4$ and $r=0.17 \mathrm{~m}, l=0.4$ and $r=0.23 \mathrm{~m}, l=0.5$ and $r=0.15 \mathrm{~m}, l=0.5$ and $r=0.25 \mathrm{~m}, l=0.6$ and $r=0.2 \mathrm{~m}$, $l=0.7$ and $r=0.1 \mathrm{~m}, l=0.8$ and $r=0.19 \mathrm{~m}$, and $l=1$ and $r=0.15 \mathrm{~m})$.

Table 3 shows the classification accuracy of the three classifiers. MPMTD gives a good result with SVM, but it is less accurate when using the other classifiers. The separation between early and late time is the weakness of all classifiers in time domain, because the automatic separation is not an easy task.

Applying MPMFD provides the best results with the three classifiers. Accuracy of $100 \%$ is reached with NB, K-NN (K $=1$ ), and SVM. In this case, the separation of the early and late time is not necessary. Moreover, with this method we have reconstructed two parts of the signal: real and imaginary parts, thus making the poles more distinctive without the need to use a large frequency band.

\section{Conclusions}

In the paper, the importance of features extraction for targets classification has been shown. The results indicate that features extracted with MPMFD present a plausible solution to automatic target classification especially for less resonant objects. With this method the separation between the early and late time is not required. A database containing object signatures can be built by using MPMFD. For resonant objects, applying MPMTD to extract CNRs is a good solution; the advantage of CNRs is their aspect independence.

In this special case of using the monostatic radar and symmetrical targets, MPMFD is a robust method. The future work will be devoted to test this method with complex objects where we need to take the angle aspect into consideration. 
TABLE 2: Features extracted by MPMFD from a thin wire $(l=3 \mathrm{~m})$, a sphere $(r=0.3 \mathrm{~m})$, and a cylinder $(l=0.6 \mathrm{~m}, r=0.2 \mathrm{~m})$ (poles $\times 10^{8}$ ).

\begin{tabular}{lccc}
\hline & Thin Wire & Sphere & Cylinder \\
\hline$P_{1}$ & $-5.096-43.80 j$ & $-0.60-2.970 j$ & $-0.642-3.443 j$ \\
$P_{2}$ & $-4.678-33.57 j$ & $-0.150-2.046 j$ & $-0.184-1.994 j$ \\
$P_{3}$ & $-2.278-24.07 j$ & $-1.013-1.108 j$ & $-0.584-1.122 j$ \\
$P_{4}$ & $-1.169-19.47 j$ & $-0.740+0.884 j$ & $0.093-0.583 j$ \\
$P_{5}$ & $-0.426-12.61 j$ & $-0.002+1.242 j$ & $0.039+0.793 j$ \\
$P_{6}$ & $-10.80-7.60 j$ & 0 & $0.003+1.014 j$ \\
$P_{7}$ & $-4.173-6.851 j$ & 0 & 0 \\
$P_{8}$ & $-0.229-6.294 j$ & 0 & 0 \\
$P_{9}$ & $0.021-0.002 j$ & 0 & 0 \\
$P_{10}$ & $-0.292+0.534 j$ & 0 & 0 \\
$P_{11}$ & $-3.656+28.76 j$ & 0 & 0 \\
$P_{12}$ & $-4.743+39.70 j$ & 0 & 0 \\
\hline
\end{tabular}

TABLE 3: Classification accuracy.

\begin{tabular}{|c|c|c|c|c|c|}
\hline \multirow{2}{*}{ Classifiers } & \multirow{2}{*}{ Naïve Bayes } & \multicolumn{3}{|c|}{ K-NN } & \multirow{2}{*}{ SVM } \\
\hline & & $K=1$ & $K=3$ & $K=5$ & \\
\hline $\begin{array}{l}\text { MPM in time } \\
\text { domain }\end{array}$ & $75 \%$ & $83.33 \%$ & $83.33 \%$ & $79.17 \%$ & $91.67 \%$ \\
\hline $\begin{array}{l}\text { MPM in frequency } \\
\text { domain }\end{array}$ & $100 \%$ & $100 \%$ & $91.67 \%$ & $91.67 \%$ & $100 \%$ \\
\hline
\end{tabular}

New canonical targets (cube, strip, etc.) and dielectric objects will be also treated to generalize the identification to new types of targets.

\section{Conflict of Interests}

The authors declare that there is no conflict of interests regarding the publication of this paper.

\section{Acknowledgment}

Gyeonggi Technology Development Program has supported this work by a Grant (I09200) funded by Gyeonggi Province.

\section{References}

[1] I. Y. Immoreev, "Ultrawideband radars: features and capabilities," Journal of Communications Technology and Electronics, vol. 54, no. 1, pp. 1-26, 2009.

[2] E. J. Rothwell and K. M. Chen, "A hybrid E-pulse/least squares technique for natural resonance extraction," Proceedings of the IEEE, vol. 76, no. 3, pp. 296-298, 1988.

[3] R. Kumaresan and D. W. Tufts, "Estimating the parameters of exponentially damped sinusoids and pole-zero modeling in noise," IEEE Transactions on Acoustics, Speech, and Signal Processing, vol. 30, no. 6, pp. 833-840, 1982.

[4] T. K. Sarkar and O. Pereira, "Using the Matrix Pencil method to estimate the parameters of a sum of complex exponentials,"
IEEE Antennas and Propagation Magazine, vol. 37, no. 1, pp. 4855, 1995.

[5] N. V. Z. Shuley, "A review of uncooperative target identification using UWB resonance based radar techniques," ECTI Transactions on Electrical Engineering, Electronics, and Communications, vol. 4, no. 2, pp. 118-126, 2006.

[6] G. Turhan-Sayan, "Real time electromagnetic target classification using a novel feature extraction technique with PCA-based fusion," IEEE Transactions on Antennas and Propagation, vol. 53, no. 2, pp. 766-776, 2005.

[7] W. Lee, T. K. Sarkar, H. Moon, and M. Salazar-Palma, "Computation of the natural poles of an object in the frequency domain using the Cauchy method," IEEE Antennas and Wireless Propagation Letters, vol. 11, pp. 1137-1140, 2012.

[8] M. Khodjet-Kesba, K. Chahine, K. El Khamlichi Drissi, and K. Kerroum, "Comparison of ultra-wideband radar target classification methods based on complex natural resonances," in Proceedings of the Progress In Electromagnetics Research Symposium (PIERS '12), pp. 306-309, Kuala-Lumpur, Malaysia, March 2012.

[9] C.-C. Chen, "Electromagnetic resonances of immersed dielectric spheres," IEEE Transactions on Antennas and Propagation, vol. 46, no. 7, pp. 1074-1083, 1998.

[10] C. E. Baum, "The singularity expansion method," Transient Electromagnetic Field, vol. 10, pp. 129-179, 1976.

[11] J. E. Mooney, Z. Ding, and L. S. Riggs, "Robust target identification in white Gaussian noise for ultra wide-band radar systems," IEEE Transactions on Antennas and Propagation, vol. 46, no. 12, pp. 1817-1823, 1998.

[12] M. McClure, R. C. Qiu, and L. Carin, "On the superresolution identification of observables from swept-frequency scattering data," IEEE Transactions on Antennas and Propagation, vol. 45, no. 4, pp. 631-641, 1997.

[13] M. P. Hurst and R. Mittra, "Scattering center analysis via Prony's method," IEEE Transactions on Antennas and Propagation, vol. 35, no. 8, pp. 986-988, 1987.

[14] K. Chahine, K. El Khamlichi Drissi, C. Pasquier et al., "Electric load disaggregation in smart metering using a novel feature extraction method and supervised classification," Energy Procedia, vol. 6, pp. 627-632, 2011.

[15] T. Mitchell, Machine Learning, McGraw-Hill, New York, NY, USA, 1997.

[16] T. Cover and P. Hart, "Nearest neighbor pattern classification," IEEE Transactions on Information Theory, vol. 13, no. 1, pp. 2127, 1967.

[17] V. Kecman, Learning and Soft Computing, MIT Press, Cambridge, UK, 2001.

[18] FEKO Comprehensive Electromagnetic Solutions, User's Manual, Suite 6.0, 2010. 

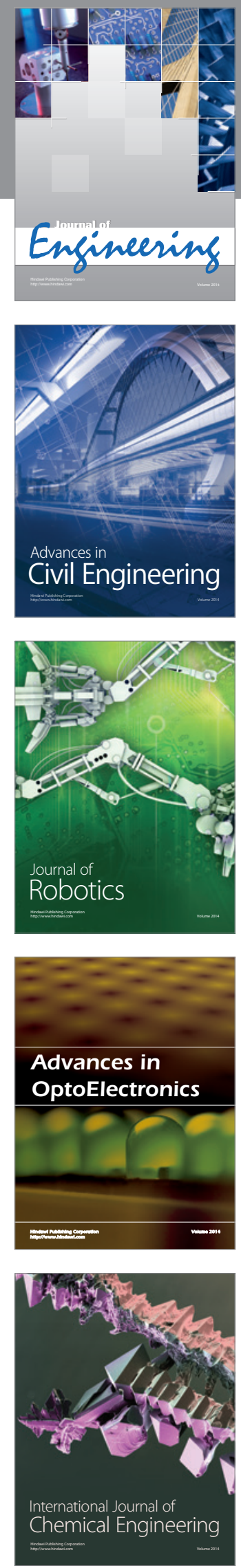

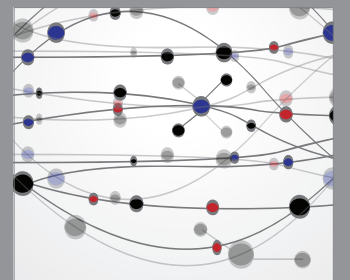

The Scientific World Journal
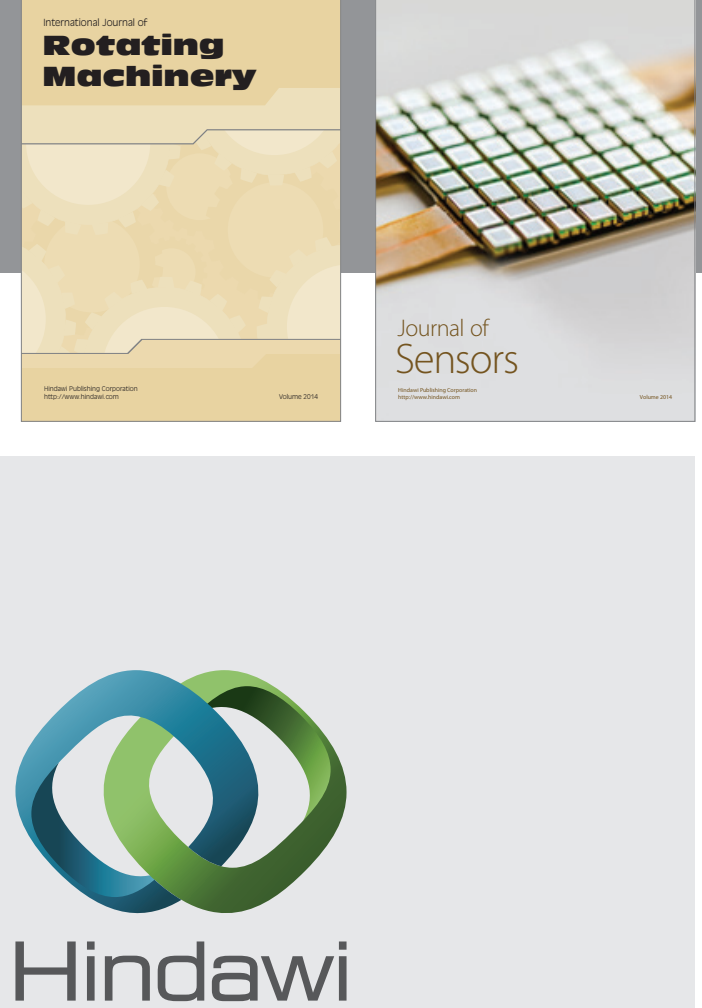

Submit your manuscripts at http://www.hindawi.com
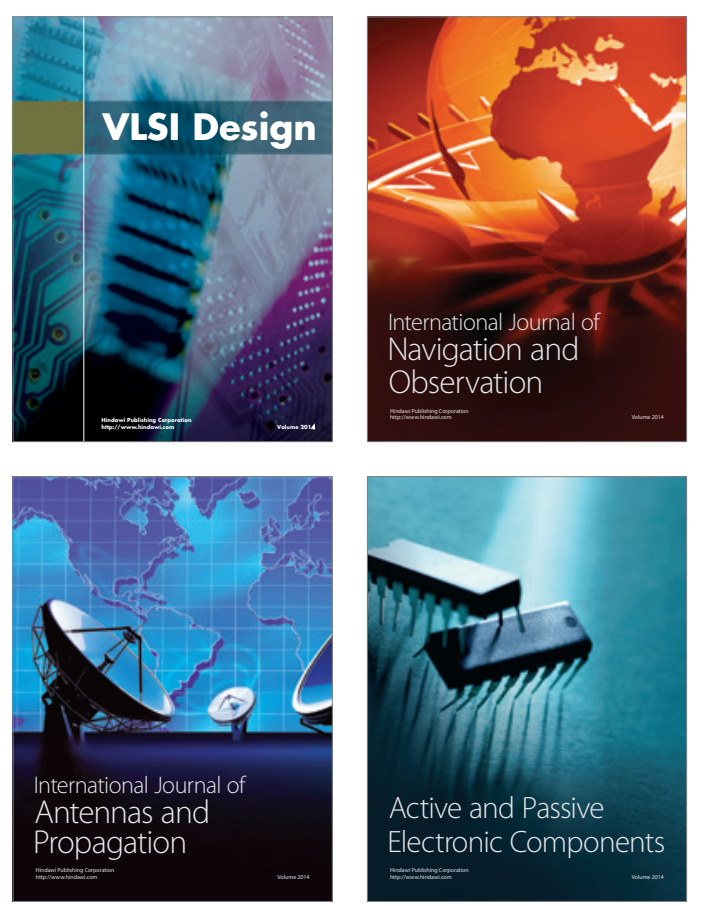
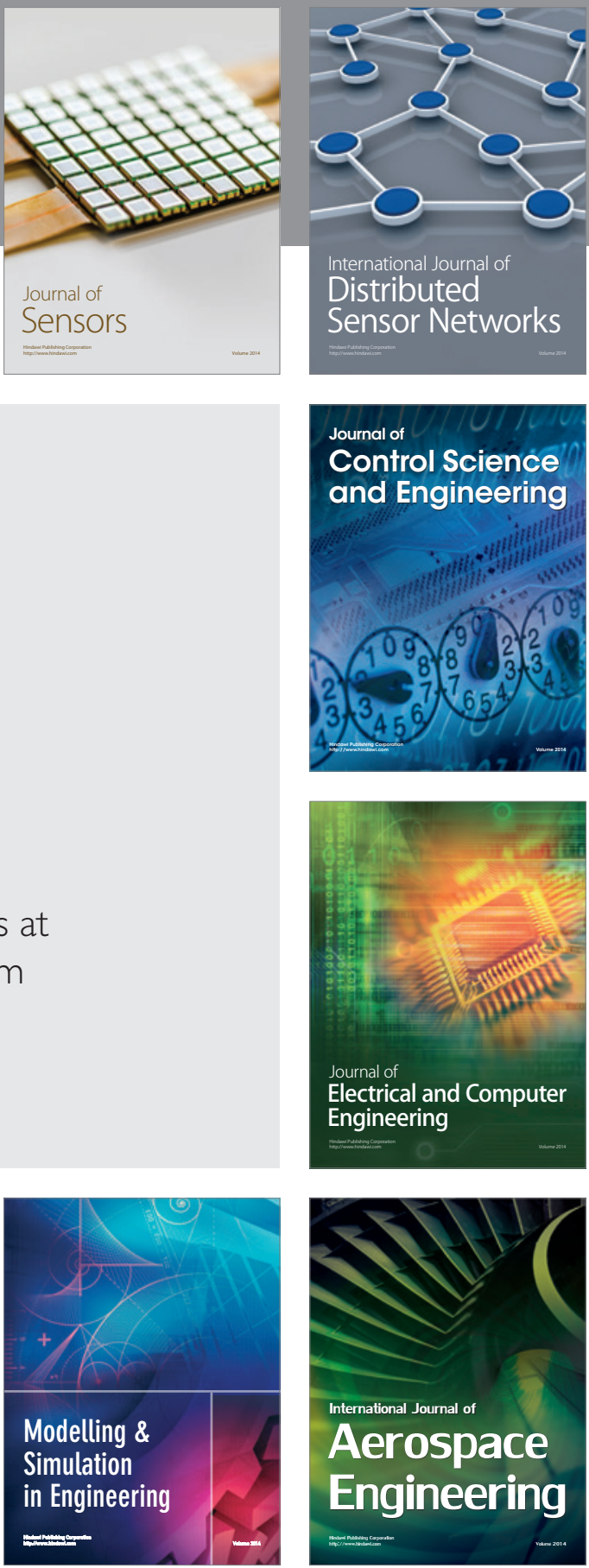

Journal of

Control Science

and Engineering
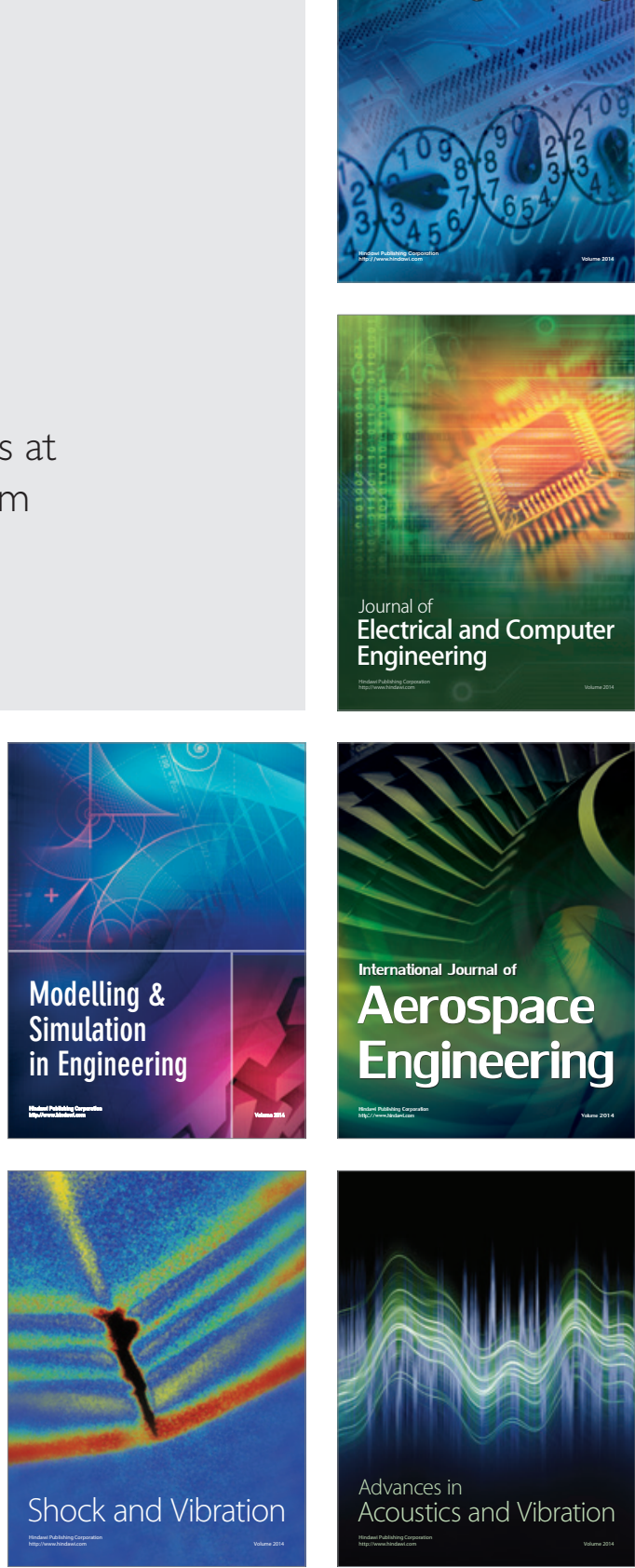